Notre Dame Journal of Formal Logic

Volume 29, Number 4, Fall 1988

\title{
On the Logic of Contingent Relevant Implication: A Conceptual Incoherence in the Intuitive Interpretation of $R$
}

\author{
MARK LANCE*
}

The primary philosophical importance of relevance logics lies in their claim to provide intuitively plausible explications of preanalytic concepts of entailment. The crucial advantage of such systems lies in the inferential role they assign to ' $\rightarrow$ '. Traditional formal systems have validated such sentences as ' $P \rightarrow(Q \rightarrow P)$ ' which, according to the relevance logician, commits both a fallacy of relevance and a fallacy of necessity. That is to say that entailment, in the sense in which that concept is operative in mathematical practice, presupposes both the relevance of antecedent to consequent and the necessity of all valid entailments. Both of these criteria are violated in the case of the above alleged entailment.

By taking both of these features into account in plausible and systematic ways, Anderson and Belnap [2] developed the system $\mathbf{E}$ of entailment which allows us to interpret coherently the symbol ' $\rightarrow$ ' as expressing entailment. That is, so interpreted, the logical truths of the system are plausibly close to our preanalytic notions of what entailments are true.

Along the way to the development of $\mathbf{E}$, Anderson and Belnap present the system $\mathbf{R}$ of relevant implications. The arrow of $\mathbf{R}$ is supposed to express an implication relation which, while maintaining relevance, does not imply necessity. But the status of the system $\mathbf{R}$ was clearly less secure than that of $\mathbf{E}$ in the minds of its inventors. The interpretation is less forthrightly presented and the system is less strongly endorsed than $\mathbf{E}$.

Recently this attitude has undergone something of a reversal within the general community of relevance logicians. Now there is a great deal of interest in $\mathbf{R}$, both formally and in application, while many have abandoned $\mathbf{E}$ in favor of

*I am very grateful to Phillip Kremer for a helpful discussion of an earlier version of this argument that cleared up several confusions for me and to an anonymous referee for this journal for a number of useful suggestions. 
a closely related system $\mathbf{R}^{\square}$. In this paper I argue that this tendency is misguided. I argue, in fact, that $\mathbf{R}$ is conceptually flawed in a serious way. This is not to say that $\mathbf{R}$ is useless for all purposes, but merely that it is not a good theory of the deductive inferential content of a connective expressing a relevant but nonnecessary implication relation. The argument for this claim will comprise Section 1 of this paper and will rely solely on motivational arguments which are central to the project of relevance logic itself. Further, by rejecting $\mathbf{R}$, we will be seen to have undercut one important argument for the rejection of $\mathbf{E}$ in favor of $\mathbf{R}^{\square}$. In Section 2, we present an alternative natural deduction system-fRI, which is a more plausible candidate for a theory of relevant nonnecessary implication - and develop an equivalent axiomatic formulation. Finally, in Section 3 we add modal rules to the system fRI and show that under the appropriate translation, this system is equivalent to $\mathbf{E}$. Since the temptation to reject $\mathbf{E}$ in favor of $\mathbf{R}^{\square}$ was due to the fact that $\mathbf{R}^{\square}$ does not turn out to be equivalent to $\mathbf{E}$ under this translation, this will provide another important motivation for fRI.

1 The argument against $\boldsymbol{R}$ Whereas the system $\mathbf{E}$ provides us with an entailment connective that takes account of both the requirement of relevance and that of necessity, the system $\mathbf{R}$ is supposed to take into account only the requirement of relevance. Thus, in [2] we read the following:

In chapter I we motivated the calculus $\mathbf{E}_{\rightarrow}$, which from one point of view is the kernal of $\mathbf{E}$, by adversion to two sets of considerations, one involving necessity and the other relevance. Now it is perfectly clear from the way chapter I goes that these two sets of considerations are separable if not independent, the former issuing as it does in the calculus $\mathbf{S} \mathbf{4}_{\rightarrow}$, whereas the latter leads us to $\mathbf{R}_{\rightarrow} \ldots$... Another advantage of studying relevance when separated from necessity is that one can be perfectly sure that one is not pigheadedly studying logical implication from what some would think a perverse point of view; for the connective of $\mathbf{R}$, like that of intuitionism, makes no claim to logical force. (pp. 349-350)

This suggests the standard reading of the arrow of $\mathbf{R}$ which is every bit as intuitive as that for the arrow of $\mathbf{E}$ (if less precisely understood). ' $\rightarrow$ ', as formalized in $\mathbf{R}$, expresses (one of, or an aspect of) the sort of nonnecessary implication relation that is common in ordinary discourse. When we claim that if the Browns do not improve their receiving corps, they will fail in the playoffs next year, we do not mean to suggest that this implication has the necessary force of logical entailment, but we do certainly imply a connection of relevance between antecedent and consequent. Some such intuitive notion is what is being formalized in $\mathbf{R}$. Such sentiments can be found throughout the writings of the most prominent relevance logicians:

E lacks a relevant, contingent sense of "if-then"; accordingly, $\mathbf{R}$ promises to have application wherever what is wanted is a conditional the meaning of which, while non-logical, involves relevance of antecedent to consequent. Since certain it is that conditional locutions in English usually do not have logical force, one would expect the arrow of $\mathbf{R}$ more often than that of $\mathbf{E}$ to be a suitable target for translation from the vernacular. ([2], p. 351) 
Now, although Anderson and Belnap hedge their bet on this interpretation with the claim that these remarks are only intended to be programmatic, others are less hesitant. Bacon [3], for example, utilizes the implication relation of $\mathbf{R}$ as the basis of a theory of subjunctive conditionals (see also [4] and [1]). Further, this intuitive interpretation is behind the argument of Routley and Meyer [6] and Anderson and Belnap [2] that $\mathbf{E}$ ought to be the modalization of $\mathbf{R}$. That is to say, if we add an $\mathbf{S} 4$ theory of modality to $\mathbf{R}$ to obtain $\mathbf{R}^{\square}$ we should have that any formula $A$ is a theorem of $\mathbf{E}$ iff the result of replacing every subformula of $A$ which is of the form $B \rightarrow C$ with $\square(B \rightarrow C)$ is a theorem of $\mathbf{R}^{\square}$.

It was assumed for some time by all concerned that this equivalence would hold. More importantly, it was claimed by Routley and Meyer (and with some hesitation by Anderson and Belnap) that should it fail to hold (as it has turned out to do), one ought to reject $\mathbf{E}$ in favor of $\mathbf{R}^{\square}$. The argument was simply that a necessary relevant conditional ought to be the necessitation of a nonnecessary relevant conditional. This argument is perfectly correct except that it relies on the mistaken presupposition that $\mathbf{R}$ is a good theory of a nonnecessary relevant conditional. By demonstrating that this is not the case, we hope to scuttle any temptation to reject $\mathbf{E}$ on these grounds.

Consider the sentence ' $A \rightarrow(B \rightarrow(A \& B))$ '. This is, of course, not a logical truth according to either $\mathbf{R}$ or $\mathbf{E}$. It could not be, for if it were added to $\mathbf{R}$ we would be able to prove $A \rightarrow(B \rightarrow A)$, which is a paradigm fallacy of relevance. But this is just an argument based on the overall utility of the whole system and does not provide a rationale for the rejection of this formula rather than some other (e.g. ' $A \& B \rightarrow B$ '). It is to be hoped that there would also be some intuitive story as to why this sentence itself is not valid. And Anderson and Belnap do provide us with such a motivation for the rejection of this sentence:

Think of examples. When it is said that the axioms of group theory imply that the identity element is unique, we understand that their conjunction implies this. No one would understand the statement as meaning that closure, associativity, and existence of an identity element conjointly imply that the-existence-of-an-inverse entails that the-identity-element-is-unique. How could we deduce anything about entailments from an incomplete set of axioms for groups, which don't even mention entailment? Similarly, no one has supposed he could deduce from the statement that Socrates is a man, that the fact that all men are mortal entails that Socrates is mortal. The statement that Socrates is a man has no consequences whatever which have to do with such essentially logical matters as entailment. ([2], p. 262)

As Anderson and Belnap point out, these considerations are very strong, ruling out even the much weaker claim that $B$ materially "implies" $A \rightarrow A \& B$, for it is possible for the antecedent to be true and the consequent to be false. Thus, 'Grass is green' does not imply 'Grass is green and Reagan is sleeping'. This is a simple and obvious truth about implication and has nothing to do with a supposition that Reagan is sleeping.

With these very convincing considerations in mind, let us switch our attention to the following sentence which is an axiom of both $\mathbf{R}$ and $\mathbf{E}:(A \rightarrow B) \rightarrow$ $((B \rightarrow C) \rightarrow(A \rightarrow C))$. One would think, on first glance, that the same argument ought to go through for this sentence. Suppose that $A, B$, and $C$ are distinct atomics. Then it is simply false that $B \rightarrow C$ entails $A \rightarrow C$. And this fact should 
hold as before quite independently of what else is merely true. Maybe it is true that $A$ implies $B$, but it is surely not part of the content of this claim that $B \rightarrow$ $C$ (all by itself) implies $A \rightarrow C$. One who was convinced by the earlier argument of Anderson and Belnap may be inclined to agree with C. I. Lewis:

I doubt whether this proposition should be regarded as a valid principle of deduction: it would never lead to any inference $A \rightarrow C$ which would be questionable when $A \rightarrow B$ and $B \rightarrow C$ are given as premises; but it gives the inference $(B \rightarrow C) \rightarrow(A \rightarrow C)$ whenever $A \rightarrow B$ is a premiss. Except as an elliptical statement for " $((A \rightarrow B) \&(B \rightarrow C)) \rightarrow(A \rightarrow C)$ and $A \rightarrow B$ is true," this inference is dubious. ([5], p. 496)

Despite the apparent similarity between this claim and the previous argument of Anderson and Belnap, the latter quote this assertion of Lewis's and reject it out of hand:

On the contrary, Ackermann 1956 is surely right that "unter der Voraussetzung $A \rightarrow B$ ist der Schluss von $B \rightarrow C$ auf $A \rightarrow C$ logisch zwingend." The mathematician is involved in no ellipsis in arguing that "if the lemma is deducible from the axioms, then this entails that the deducibility of the theorem from the axioms is entailed by the deducibility of the theorem from the lemma." ([2], p. 9)

But why is the mathematician involved in no ellipsis? How could we possibly deduce anything concerning entailments between claims about entailment given merely the premise, for example, that a lemma about groups follows from the axioms of group theory? Surely a claim about an inferential connection between two sentences of the language of group theory tells us nothing about inferential relations between sentences containing the fundamentally logical concept of implication or entailment. ${ }^{1}$

The issue is, of course, closely connected to that of the correct formulation of the natural deduction rule reiteration and it is by consideration of the proper use of this rule that we come to see the real disanalogy between the two cases. If we are attempting to deduce $B$ on the $\operatorname{single}$ assumption $A$, we are not allowed to make use in our derivation of other random truths. If we could, we could make use of the fact that snow is white to derive the claim that snow is white "from" the claim that Reagan is sleeping. Thus we should not allow ourselves to import established truths willy-nilly into subproofs.

But we need not restrict all such importations either. This very point is made by Anderson and Belnap in their discussion of the natural deduction rule of reiteration:

After the word "Proof:" in a mathematical treatise, mathematical writers seem to feel that no more hypotheses may be introduced; and it is regarded as a criticism of a proof if not all the required hypotheses are stated explicitly at the outset. Of course additional machinery may be invoked in the proof, but this must be of a logical character, i.e., in addition to the hypotheses, we may use in the argument only propositions tantamount to statements of logical necessity. These considerations suggest that we should be allowed to import into a deduction (i.e., into a subproof by reit) only propositions which, if true at all, are necessarily true: i.e., we should reiterate only entailments. ([2], p. 15) 
This then presents the relevant disanalogy between the two cases. In the case of $A \rightarrow(B \rightarrow(A \& B))$ the premise may only be contingent whereas in the case of the transitivity law, the antecedent is an entailment. The objective to this response should by now be obvious, however. The distinction, although perfectly good and to the point in the context of $\mathbf{E}$, is irrelevant in the context of $\mathbf{R}$, for there $A \rightarrow B$ may only be contingent.

In the formulation of $\mathbf{E}$, Anderson and Belnap take account of the fact that only necessary truths should be allowed into subproofs by way of reiteration and thus restrict the rule to sentences of the form $A \rightarrow B$. In $\mathbf{R}$, however, in virtue of the fact that this system is aiming only to capture features of relevance, no restriction is placed on reiteration. But this does not fit with the intuitive motivation or interpretation of the arrow in the context of $\mathbf{R}$. If implications in $\mathbf{R}$ are meant only to imply contingent relations (and if the aim of a logical system is to characterize the set of logically valid entailments involving sentences which contain certain bits of vocabulary) then we should not put fewer restrictions on reiteration, but more. Since there are no sentences in $\mathbf{R}$ which must be necessarily true if true at all, we should not allow reiterations of any of them. To do so is simply to infer fallaciously by Anderson and Belnap's own argument. ${ }^{2}$

One might claim that $\mathbf{R}$ is meant to capture only features of relevance and not those of necessity, and mean this in a different sense than that in which we have meant it so far. One might argue that classical logic allows us to commit two sorts of fallacies: fallacies of relevance and fallacies of necessity. $\mathbf{E}$ is the system one gets when all fallacies are ruled out. In $\mathbf{R}$ we allow some fallacies those involving only necessity - while ruling out those involving relevance.

Aside from the fact that this line would rob $\mathbf{R}$ of much of the intuitive interest it had when we were taking it to actually explicate the correct logicalinferential content of a type of conditional that was useful and important, there is a more pressing internal criticism of this line. If this is the motivation for $\mathbf{R}$ then we lose our justification for the rejection of ' $A \rightarrow(B \rightarrow(A \& B))$ ' in $\mathbf{R}$, for this formula also commits only a fallacy of necessity. Thus, depending on how we interpret $\mathbf{R}$, it has either too many or too few theorems.

This interpretation of the point of $\mathbf{R}$ seems misguided in any event. When we develop a logical system we are attempting to capture the deductive inferential content of certain bits of vocabulary; that is, we are trying to spell out the valid entailments which hold between sentences involving that vocabulary. Thus, a theory of nonnecessary relevant implication should not try to determine the nonnecessary relevant implication relations that hold between sentences involving the connective - a job that presumably falls to epistemology or philosophy of science rather than logic-but rather should give us the entailment relations that hold between such sentences.

These considerations should remove the temptation to reject $\mathbf{E}$ in favor of $\mathbf{R}^{\square}$. If $\mathbf{R}$ does not even capture the correct deductive inferential content of a nonnecessary relevant conditional, we have no reason to think that its necessitation would produce an entailment connective. But we still do have strong reason to want some system of nonnecessary relevant implication and if $\mathbf{E}$ is a correct theory of entailment, the necessitation of this conditional should be equivalent to E. We turn now to the system RI. In Section 3, we show that the necessitation of the conditional so codified is equivalent to the conditional of $\mathbf{E}$. 
2 The system RI One criticism of a system which disallows reiteration altogether is that it seems to become trivial. Indeed, the only theorems of the pure implicational fragment of such a system are the substitution instances of $A \rightarrow A$. But the theorems of the implicational fragment of the system do not exhaust the inferential content of the connective in question for they correspond only to inferences with only one premise. If we allow inferences with two premises we see that such patterns as transitivity - from $A \rightarrow B$ and $B \rightarrow C$ to infer $A \rightarrow C$-ought also to be included as valid.

When we include other vocabulary the matter becomes more interesting still. Not only do we have the above inference rule which corresponds to the single axiom $((A \rightarrow B) \&(B \rightarrow C)) \rightarrow(A \rightarrow C)$, but the interaction of conjunction with the conditional will lead us to expect $((A \rightarrow B) \&(A \rightarrow C)) \rightarrow(A \rightarrow B \&$ $C)$. A similar axiom governing the interaction of the conditional and disjunction will be included as well.

We assume a language including $\&, \vee, \sim$, and $\rightarrow$. We present a Fitch-style formulation of our proposed theory of relevant implication and dub it 'fRI'. We will follow the subscripting and grammatical conventions of [2] without further explanation. fRI is defined by the following natural rules:

Hyp A step may be introduced as the hypothesis of a new subproof, and each new hypothesis receives a unit class $\{k\}$ of numerical subscripts, where $k$ is the rank (see [2], §8.1).

Rep $A_{a}$ may be repeated, retaining relevance indices $a$.

CP From a proof of $B_{a}$ on hypothesis $A_{\{k\}}$ to infer $A \rightarrow B_{a-\{k\}}$, provided $k \epsilon a$.

MPP From $A_{a}$ and $A \rightarrow B_{b}$ to infer $B_{a \cup b}$.

Red From $A \rightarrow \sim A_{a}$ to infer $\sim A_{a}$.

MTT From $A \rightarrow B_{a}$ to infer $\sim B \rightarrow \sim A_{a}$.

vI From $A_{a}$ to infer $A \vee B_{a}$. From $B_{a}$ to infer $A \vee B_{a}$.

$\vee \rightarrow \quad$ From $A \rightarrow C_{a}$ and $B \rightarrow C_{a}$ to infer $(A \vee B) \rightarrow C_{a}$.

[Note: This rule implies the rule $\vee \mathbf{E}$ : From $A \vee B_{a}, A \rightarrow C_{b}$ and $B \rightarrow C_{b}$ to infer $C_{a \cup b}$.]

Trans From $A \rightarrow B_{a}$ and $B \rightarrow C_{b}$ to infer $A \rightarrow C_{a \cup b}$.

$\& \rightarrow \quad$ From $A \rightarrow B_{a}$ and $A \rightarrow C_{a}$ to infer $A \rightarrow(B \& C)_{a}$.

\&I From $A_{a}$ and $B_{a}$ to infer $A \& B_{a}$.

\& $\mathbf{E}$ From $A \& B_{a}$ to infer $A_{a}$. From $A \& B_{a}$ to infer $B_{a}$.

$\& \vee \quad$ From $A \&(B \vee C)_{a}$ to infer $(A \& B) \vee C_{a}$.

DNI From $A_{a}$ to infer $\sim A_{a}$.

DNE From $\sim A_{a}$ to infer $A_{a}$.

The system fRI has, I believe, a good claim to provide the correct deductive inferential theory of a contingent relevant conditional. The theorems of fRI are clearly a subset of $\mathbf{R}$ since every rule of fRI is provable in $\mathbf{R}$. But the system does not fail to give an interesting inferential content to the arrow since it is easy to show that if $A \rightarrow(B \rightarrow C)$ is provable in $\mathbf{R}$, then $(A \& B) \rightarrow C$ is provable in fRI. Thus, if we are correct in out amplification of Lewis's claim that the intuitive motivation for the former is, in general, derivative upon a confusion with the latter, then fRI preserves all the intuitive content of relevant implication which was miscodified in $\mathbf{R}$. 
Since fRI is a subsystem of $\mathbf{R}$, no paradoxes of relevance are provable in fRI. Thus fRI can be supported by all the negative arguments which Anderson, Belnap, and cohorts bring to bear against classical systems. Further, since by adding a normal rule of reiteration to fRI we would obtain $\mathbf{R}, \mathbf{f R I}$ is supported by the positive motivations which Anderson and Belnap bring forward for the restrictions on this rule in the context of $\mathbf{E}$. A final virtue of $\mathbf{f R I}$ is demonstrated in Section 3 where it is shown that its necessitation is equivalent to E. For now, however, we turn to the axiomatic formulation of fRI.

The system RI will be defined by the following axioms and rules:

A1 $A \rightarrow A$

A2 $A \&(A \rightarrow B) \rightarrow B$

A3 $(A \rightarrow \sim A) \rightarrow \sim A$

A4 $(A \rightarrow B) \rightarrow(\sim B \rightarrow \sim A)$

A5 $\quad((A \rightarrow C) \&(B \rightarrow C)) \rightarrow((A \vee B) \rightarrow C)$

A6 $((A \rightarrow B) \&(A \rightarrow C)) \rightarrow(A \rightarrow(B \& C))$

A7 $((A \rightarrow B) \&(B \rightarrow C)) \rightarrow(A \rightarrow C)$

A8 $A \rightarrow \sim \sim A$

A9 $\sim A \rightarrow A$

$\mathbf{A 1 0}(A \& B) \rightarrow A$

A11 $(A \& B) \rightarrow B$

A12 $A \rightarrow(A \vee B)$

A13 $A \rightarrow(B \vee A)$

A14 $A \&(B \vee C) \rightarrow(A \& B) \vee C$

R1 $A \rightarrow B \vdash(B \rightarrow C) \rightarrow(A \rightarrow C)$

R2 $B \rightarrow C \vdash(A \rightarrow B) \rightarrow(A \rightarrow C)$

R3 $A, A \rightarrow B \vdash B$

R4 $A, B \vdash A \& B$.

It is quite easy to prove the equivalence of RI and fRI. To show that RI is a subsystgem of fRI we only need show that A1-A13 are theorems of fRI and that R1-R4 are valid in fRI. This much is trivial. To show that fRI is a subsystem of RI we use the method of quasi-proofs of [2], $\$ 4.1$. The proof is much easier here, however, since we do not even need the lemma utilized by Anderson and Belnap. The important point is that there is no rule of reiteration in fRI, so any formula within an innermost subproof will have either $\varnothing$ or $\{k\}$ as relevance index where $\{k\}$ is the index of the hypothesis of that subproof. This greatly facilitates the demonstration that each step of the quasi-proof is verifiable. The details of the proof are left to the reader.

$3 \boldsymbol{R I}$ and $\boldsymbol{E}$ We turn finally to the task of showing that the necessitation of the conditional of $\mathbf{R I}$ is equivalent to the conditional of $\mathbf{E}$. We define $\mathbf{f R I} \mathbf{I}^{\square}$ to be the system obtained by adding to fRI the following modal rules:

$\square \mathbf{E}$ From $\square A_{a}$ to infer $A_{a}$.

$\square \rightarrow$ From $\square(A \rightarrow B)_{a}$ to infer $(\square A \rightarrow \square B)_{a}$.

$\square \&$ From $(\square A \& \square B)_{a}$ to infer $\square(A \& B)_{a}$.

$\square 4$ From $\square A_{a}$ to infer $\square \square A_{a}$.

Reit $A_{a}$ may be reiterated (retaining subscripts) into hypothetical subproofs provided $A$ has the form $\square B$. 
It must be remarked that our claim that the resultant system is equivalent to $\mathbf{E}$ under the relevant translation is not entirely analogous to the original hoped-for equivalence of $\mathbf{R}^{\square}$ and $\mathbf{E}$. In the latter case we could consider an axiomatic formulation of $\mathbf{R}$ and hope to add natural $\mathbf{S 4}$ axioms to it so as to produce an axiomatic theory of entailment. In the present case, we are not dealing with axiomatic theories at all and it is not simple to transform our natural deduction account of modality into an axiomatic theory with RI as the background logic. At least the set of axioms will not be the normal ones for S4. So in a sense we are after a weaker goal than were those who attempted to prove the equivalence of $\mathbf{R}^{\square}$ and $\mathbf{E}$. Nonetheless, the natural deduction formulation of all such systems provides far more by way of semantic explication of the concepts involved than does the axiomatic theory, and the natural deduction rules given above are no doubt the most natural.

In order to prove the equivalence of $\mathbf{f R I} \square$ and $\mathbf{E}$ we need first to define a translation from the language of $\mathbf{E}$ to that of $\mathbf{f R I} \mathbf{I}^{\square}$. Let $t(A)$ be the result of replacing every subformula of $A$ which is of the form $B \rightarrow C$ with the formula $\square(B \rightarrow C)$. We now show that $\vdash_{\mathbf{E}} A$ iff $\vdash_{\mathbf{f R I}} \square t(A)$. The proof is, in fact, quite easy. Let $t^{*}(A)$ be the result of replacing every subformula of $A$ which is of the form $\square B$ with the formula $(B \rightarrow B) \rightarrow B$. This is the formula that defines $\square B$ in $\mathbf{E}$ (see [2], §4.3). We will think of this as our translation from fRI ${ }^{\square}$ back to $\mathbf{E}$, but note that $t^{*} \neq t^{-1}$.

We first show that if $t(A)$ is provable in $\mathbf{f R I} \mathbf{I}^{\square}$ then $A$ is provable in $\mathbf{E}$. Suppose that we are given a proof in $\mathbf{f R I} \square$ of $t(A)$. Let us consider the same sequence of steps, subscripts, and subproof indications with $t^{*}(t(A))$ replacing $t(A)$ at each step. Each of the rules of fRI except Trans are rules of $\mathbf{E}$ and remain applicable under the $t^{*}$ transformation. Further, Trans is easily shown valid in $\mathbf{E}$ and remains applicable under the $t^{*}$ transformation. It is shown in [2] that each of RN, $\square \mathbf{E}, \square \rightarrow, \square \&$, and $\square 4$ are valid in $\mathbf{E}$ with the $t^{*}$ transformation. Finally, any application of Reit will remain valid since $t^{*}(\square B)$ is a conditional. Thus each step of our proof is valid in $\mathbf{E}$ and we therefore have a proof of the transformation.

What, then, is our proof a proof of? Since we have applied both $t$ and $t^{*}$ to $A$, what we have is the result of replacing every subformula of $A$ which is of the form $B \rightarrow C$ with $((B \rightarrow C) \rightarrow(B \rightarrow C)) \rightarrow(B \rightarrow C)$. But in $\mathbf{E}$ we have $(B \rightarrow$ $C) \leftrightarrow(((B \rightarrow C) \rightarrow(B \rightarrow C)) \rightarrow(B \rightarrow C))$ and substitutivity of coentailing propositions as is proved in [2]. Thus we know that we can turn our proof of $t^{*}(t(A))$ into a proof of $A$.

We now need to show that if $A$ is provable in $\mathbf{E}$ then $t(A)$ is provable in fRI ${ }^{\square}$. In this direction, it is convenient to utilize the axiomatic formulation of E. On page 340 of [2], $\mathbf{E}$ is characterized by sixteen axioms together with the rules R1: From $A$ and $B$ to infer $A \& B$; and R2: From $A \rightarrow B$ and $A$ to infer $B$. The axioms of $\mathbf{E}$ are as follows:

$$
\begin{array}{ll}
\text { E1 } & A \rightarrow A \\
\text { E2 } & (A \rightarrow B) \rightarrow((B \rightarrow C) \rightarrow(A \rightarrow C)) \\
\text { E3 } & (A \rightarrow B) \rightarrow((C \rightarrow A) \rightarrow(C \rightarrow B)) \\
\text { E4 } & (A \rightarrow(A \rightarrow B)) \rightarrow(A \rightarrow B) \\
\text { E5 } & A \& B \rightarrow A
\end{array}
$$




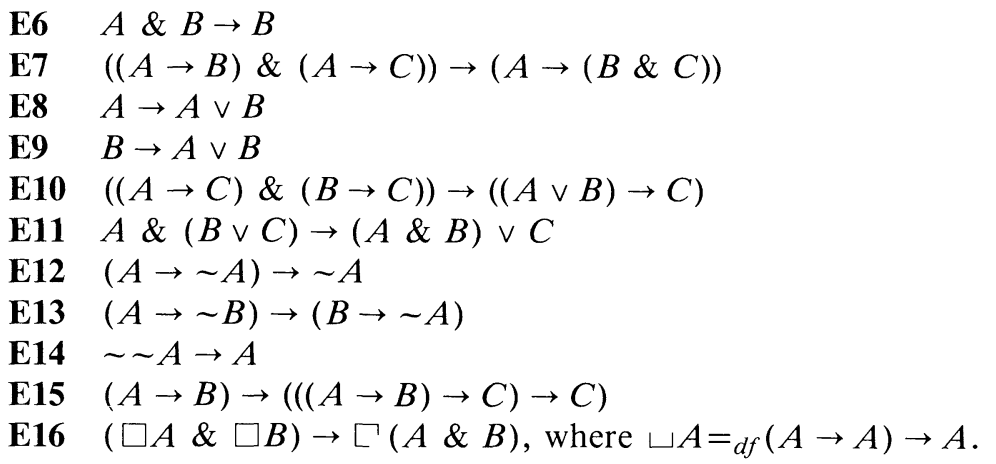

It is straightforward to verify that for each of the sixteen axioms of $\mathbf{E}$ their translation under $t$ is provable in $\mathbf{f R I}{ }^{\square}$. Now suppose we have a proof of $A$ in E. That is, we have a sequence of sentences, each of which is either an axiom or follows from earlier steps by $\mathrm{R} 1$ or $\mathrm{R} 2$. To turn this into a proof of $t(A)$ in fRI ${ }^{\square}$, we first replace each occurrence of an axiom with a proof of the $t$-transform of that axiom in $\mathbf{f R I} \square$ and each nonaxiom $A$ with $t(A)$. We now notice that the first application of an E-rule in our altered proof will not involve premises which have any subscripts. Hence, if it is an application of $\mathrm{R} 1$, it will have become a move from $t(A)_{\varnothing}$ and $t(B)_{\varnothing}$ to $t(A \& B)_{\varnothing}$. But the latter is just $t(A) \& t(B)_{\varnothing}$. Thus the move is valid in fRI ${ }^{\square}$. If, on the other hand, the first application of an E-rule is an application of $\mathrm{R} 2$, it will be a move from $t(A)_{\varnothing}$ and $t(A \rightarrow B)_{\varnothing}$ to $t(B)_{\varnothing}$. Now $t(A \rightarrow B)$ is $\square(t(A) \rightarrow t(B))$, so we will insert the step $t(A) \rightarrow t(A)_{\varnothing}$ immediately before $t(B)_{\varnothing}$. This step is justified by $\square \mathbf{E}$ and its insertion makes the application of R2 valid in $\mathbf{f R I}{ }^{\square}$. In neither case did any steps acquire subscripts, so this process can be repeated until we have turned the proof of $A$ in $\mathbf{E}$ into a proof of $t(A)$ in $\mathbf{f R I}$.

\section{NOTES}

1. Indeed, at the risk of running afoul of Anderson and Belnap's carefully crafted blurring of the distinction, we can say that $A \rightarrow B$, in the case in which $A$ and $B$ are atomic, does not mention entailment any more than an axiom of group theory; it merely uses the concept. In general a sentence, although it has an inferential content, does not imply anything about its inferential content. The particular case of sentences involving the arrow does involve implications concerning inferential content, but $A \rightarrow B$ tells us something about the inferential content of the vocabulary occurring in $A$ and $B$. If $A$ and $B$ are atomic, this claim tells us nothing about the inferential content of ' $\rightarrow$ '. On the other hand $(A \rightarrow B \rightarrow(B \rightarrow C)$ does claim something about the inferential content of ' $\rightarrow$ ', namely, that $A \rightarrow B$ implies $B \rightarrow C$.

2. It is not uncommon to hear the worry that the theory of entailment cannot possibly have theorems such as $(A \rightarrow B) \rightarrow((B \rightarrow C) \rightarrow(A \rightarrow C))$ which are not theorems of the theory of nonnecessary relevant implication, for the connective of the former is strictly stronger than that of the latter. This is mistaken, however, for in the sentence in question, not only is the main connective stronger, but the antecedent of the conditional is as well. By recognizing this fact we should cleanse our mind of all a priori presuppositions as to which logic should be "stronger". 


\section{REFERENCES}

[1] Anderson, A. R., "Some nasty problems in the formal logic of ethics," Noûs, vol. 1 (1967), pp. 345-360.

[2] Anderson, A. R. and N. D. Belnap, Jr., Entailment: The Logic of Relevance and Necessity, Vol. 1, Princeton University Press, Princeton, New Jersey, 1975.

[3] Bacon, J., "The subjunctive conditional as relevant implication," Philosophia, vol. 1 (1971), pp. 61-80.

[4] Barker, J. A., A Formal Analysis of Conditionals, Southern Illinois University Monographs, Humanities Series, No. 3, Carbondale, Illinois, 1969.

[5] Lewis, C. I. and C. H. Langford, Symbolic Logic, The Century Company, New York and London, 1939; 2nd Ed., Dover, New York, 1959.

[6] Routley, R. and R. K. Meyer, "The semantics of entailment-II," Journal of Philosophical Logic, vol. 1 (1972), pp. 53-73.

Department of Philosophy

University of Pittsburgh

Pittsburgh, Pennsylvania 15260
Department of Philosophy

Syracuse University

541 Hall of Languages

Syracuse, New York 13244-1120 